\title{
SEMI-BLIND SIGNAL SEPARATION AND CHANNEL ESTIMATION IN MIMO COMMUNICATION SYSTEMS BY TENSOR FACTORIZATION
}

\author{
Bahador Makki Abadi ${ }^{1,2}$, Amir Sarrafzadeh ${ }^{3}$, Delaram Jarchi ${ }^{1}$, Vahid Abolghasemi ${ }^{1}$ and Saeid Sanei ${ }^{1}$ \\ ${ }^{1}$ Centre of Digital Signal Processing, School of Engineering, Cardiff University, Wales, UK \\ ${ }^{2}$ Electrical Engineering Department, Islamic Azad University, Ashtian Branch, Ashtian, IRAN \\ ${ }^{3}$ Department of Communications Engineering, IRAN University of Science and Technology, IRAN
}

\begin{abstract}
In this paper, we introduce a tensor-factorization method for signal detection in MIMO applications. We address the detection problem through a 3-way tensor analysis. We represent the $4 \times 4$ MIMO received signals as a third-order tensor with modes: receiver antennas, user data symbols at each packet, and finally number of packets. Then, we demonstrate that by multi-way analysis using PARAFAC 2 we can successfully solve the blind MIMO signal detection problem. In order to solve the permutation and scaling ambiguities of the detected signals we used different $\mathrm{M}$-Sequence training symbols are used. For evaluating the method we compared our $B E R$ results with those of MMSE-VBLAST signal detection method.
\end{abstract}

Index Terms - Tensor Factorization, PARAFAC2, MIMO Detection, Fading Channel Estimation, Semi-Blind Source Separation, M-Sequence, MMSE-VBLAST.

\section{INTRODUCTION}

Multiple-input-multiple-output (MIMO) systems, with multiple antennas deployed at both the transmitter and receiver, promise improved performance and bandwidth efficiency compared with conventional systems [1]. MIMO and smart antenna systems are now widely employed to combat the problems of multi-user interference, fading in wireless channels, and to achieve high data rates. Moreover, such techniques tend not to use the information in the unknown data symbols to improve channel estimation. Semi-blind techniques can potentially enhance the quality of such estimates by making a more complete use of the available data. With few known training symbols, such techniques can avoid convergence problems associated with the blind techniques. Some work on semi-blind techniques has been reported earlier for the design of fractional semi-blind equalizers for MIMO channels [2]. There are some traditional semi-blind training-based MIMO detection and channel estimation methods. On these methods the transmitted signals involve data sequences, which are known to both the transmitter and receiver and these information can be used to channel estimation or permutation removal from blindly estimated sources.
The success of this operation is subject to the channel properties not changing during the transmission of packets that contain the training sequence and the message. This requires the quasi-static behavior of the channel [3]. In this paper we used $\mathrm{m}$-sequence training sequences to find and mitigate the permutation and scaling ambiguities for blindly estimated sources. Parallel factor analysis (PARAFAC) [4] is a well known method for factorizing a multi-way data. Some PARAFAC based MIMO receivers have been developed for code-division multiple access (DS-CDMA) systems [5][6]. In these systems PARAFAC is used to decompose a three-way tensor of the received data (e.g with three modes of spreading diversity-temporal diversity-space diversity) for channel estimation and users data detection. In conventional MIMO applications without using CDMA, the received data is a two-way data with modes of antenna and temporal samples.

Let $S=\left[\mathbf{s}_{1}, \ldots . \mathbf{s}_{n_{T}}\right]^{t}$ denote the transmitted signal where $(.)^{t}$ is transpose operation, then the corresponding received signal vector $X=\left[\mathbf{x}_{1}, \ldots, \mathbf{x}_{n_{R}}\right]^{t}$ is given by

$$
X=H S+E
$$

where, $E=\left[\mathbf{n}_{1}, \ldots, \mathbf{n}_{n_{R}}\right]^{t}$ represents white Gaussian noise matrix observed at the receiving antennas whose rows have zero mean and variance $\sigma_{n}^{2}$. The $n_{R} \times n_{T}$ channel matrix $H$ contains uncorrelated complex Gaussian fading gains. Each entry, $h_{i j}$ is a fading gain between the transmitting antenna $j$ and the receiving antenna $i$.

Having quasi-static behavior of channel $H$ we can divide the temporal mode to a limited number of packets passing through a relatively fixed fading channel. With this assumption we have a three-way tensor with (antenna, packet samples, packets) modes and therefore, any tensor factorization scheme can be applied to this data to find the common components of the tensor. PARAFAC2 factorization supports more variability in one mode of the tensor and this results in having more accurate factorization performance rather than that achieved by the traditional PARAFAC method. The reminder of the paper is structured as follows. In Section 2, the tensor factorization methods (PARAFAC-PARAFAC2) are described. In Section 3, our PARAFAC2 based MIMO detection 
and channel estimation is introduced. In Section 4 we compare our method with MMSE-VBLAST detection method and the simulations and results are provided. Finally, Section 5 concludes the paper.

\section{PARAFAC AND PARAFAC2}

PARAFAC [4]model which is used to decompose trilinear data sets with a unique solution, is given below:

$$
X_{i j k}=\sum_{r=1}^{R} H_{i r} F_{j r} C_{k r}+E_{i j k}
$$

where $X_{i j k}$ represents the $i, j, k$-th element in the three-way data set, $R$ is the number of components in common to the three modes, $H_{i r}, F_{j r}$ and $C_{k r}$ are the elements in $H, F$ and $C$ used to obtain the $X_{i j k}$ elements, and $E_{i j k}$ is the residual term. Using tensor notations, the above equation can be presented as:

$$
X_{k}=F D_{k} H^{t}+E_{k}
$$

for $k=1, \ldots, K$, where $(.)^{t}$ refers to transpose operation and $X_{k}$ represents the transposed $k$ th frontal slice of the three-way array $X, H$ and $F$ are the component matrix in the first and second mode, respectively. $D_{k}$ is a diagonal matrix, whose diagonal elements correspond to the $k$ th row of the third component matrix $C$. Finally, $E_{k}$ contains the error terms corresponding to the entries in the $k$ th frontal slice. While direct fitting is applied to the raw data, indirect fitting is applied on covariance matrices of data slices. PARAFAC direct fitting includes an alternative least squares (ALS) optimization method for obtaining $H, F$, and $D_{k}$ for all $k=1, \ldots, K$, and consequently finding the three matrices $H, F$ and $C$ of equation (2) respectively [4].

PARAFAC2 as an extension of PARAFAC is designed to deal with non-trilinear data sets, while keeping uniqueness in the solutions, as the PARAFAC model does. To do so, PARAFAC2 allows a certain freedom in one variable mode. To keep uniqueness in the solutions, all cross-product matrices $X_{k} X_{k}^{t}$ are forced to be constant over $k$, i.e. $X_{1} X_{1}^{t}=$ $X_{2} X_{2}^{t}=X_{k} X_{k}^{t}$. A similar equation in matrix notation for PARAFAC2 is given as:

$$
X_{k}=F_{k} D_{k} H^{t}+E_{k}
$$

subject to $F_{k}^{t} F_{k}=\Phi, k=1, \ldots K$ where $F_{k}$ is the component matrix in the second mode corresponding to the $k$ th frontal slice, $\Phi$, which is the matrix product of $F_{k}$ and its transpose, is required to be invariant for all slices $k=1, \ldots, K$. A direct method for fitting PARAFAC2 model is proposed by Kiers [7] and in this method by defining new variables we need to minimize

$$
\sigma\left(P_{1}, \ldots, P_{k}, F, H, D_{1}, \ldots, D_{k}\right)=\sum_{k=1}^{K}\left\|X_{k}-P_{k} F D_{k} H^{t}\right\|^{2}
$$

over all its arguments subject to the constraints $P_{k}^{t} P_{k}=I_{R}$ and $D_{k}$ diagonal, $k=1, \ldots, K$, where $I_{R}$ is an $(R \times R)$ identity matrix. To minimize this function, an ALS algorithm that alternately minimizes equation (5) over $P_{k}$ for fixed $F, D_{k}$ and $H$ for $k=1, \ldots, K$, and minimizes $F, D_{1}, \ldots, D_{K}$ and $H$ for fixed $P_{1}, \ldots, P_{K}$ is used.

After convergence, for factorizing a three-way data $X(I \times$ $J \times K)$ with $R$ common factors there are matrix outputs as: 1- $H(I \times R)$ related to the first dimension of $X$; in our application it is related to the scaled and permuted version of MIMO fading channel.

2- $P(J \times R)$ related to second dimension of $X$; in our application it is related to temporal samples in each packet.

3- $C(K \times R)$ related to third dimension of $X$ with each row equal to diagonal elements of $D_{k}$. Physically each diagonal element of $D_{k}$ shows the power of the symbols for the related source in $k$ th packet.

4- $F(R \times R)$ as a constant matrix; in our application it is related to covariance matrix of the transmitted signals.

Unlike PARAFAC which has only one $P(J \times R)$ related to its second dimension, there are $K P(J \times R)$. By this variation the residual error of PARAFAC2 decomposition is lower than the PARAFAC and better signal or factor reconstruction will be achieved for signal separation applications.

\section{PARAFAC2 BASED MIMO DETECTION}

Regarding PARAFAC2 decomposition and its outputs for each 3-D data $X(I \times J \times K)$ with $R$ factors there are $H \in R_{I \times R}, K$ number of $F \in R_{J \times R}$ and $C \in R_{K \times R}$ as outputs. If this 3-D $X$ data is the output of temporal segmentation for a 2-D mixed data then $I$ will be the number of electrodes which measure the mixed signals, $J$ will be the number of samples in each temporal segments, and $K$ will be number of segments. The outputs of PARAFAC2 decomposing this signal will be $H(I \times R)$ which includes mixing matrix for $R$ sources and the $P_{k} F$ matrices include scaled version of the separated samples in each segment. Finally $C$ includes the scaling factor for each segment. The estimated sources can be calculated from these information. By using PARAFAC2 decomposition, both the sources and the mixing matrix are estimated simultaneously during each iteration of optimization.

Similarly, PARAFAC2 based source separation method can be used for MIMO signal detection and channel estimation. In this paper we use a two-way received signal which includes two modes of antenna and temporal samples. Having a quasi-static behavior for the channel at a certain time, We can divide the temporal mode to a limited number of packets passing through a relatively fixed fading channel. With this assumption now we have a three-way tensor of $X$ with (antenna-packet symbols-packet) modes and then we use tensor factorization to decompose this tensor to find an estima- 
tion of the transmitted sources followed by channel estimation. PARAFAC2 blindly estimates the transmitted sources and also channel subject to permutation and also scaling ambiguities. On semi-blind channel estimation method some a priori information about the sources or channel are used to improve the performance of the estimation or permutation ambiguity removal technique. We used m-sequence (pseudorandom binary sequence) training sequences as pseudo information attached to the sources to solve permutation and scaling ambiguity problem on the estimated sources. At this work, Our assumptions are: 1- The number of receiver antennas are equal or greater than the number of transmitter antennas. 2The channel is selected as a flat fading channel and it can be considered quasi-static for certain duration (e.g. during sending 4 packets with a certain size). 3- The transmitted symbols of each transmitter are independent of other transmitter symbols. 4- Transmitted symbols are output of a QPSK modulator.

Having QPSK output symbols helps us to add some constraints to have an effective optimization process. All QPSK symbols always have absolute value of $\sqrt{2}$ and can be scaled to have a unit absolute value. By this assumption we are able to add two constraints to PARAFAC2 based optimization. First, all $P_{k}$ signal samples have a unit absolute value and second, all $D_{k} \mathrm{~s}$ are identity matrices.

Adding second constraint gives us a fix $C$ matrix with all one entries in all iterations. Similar to PARAFAC2 fitting we compute $P_{k}$ at the presence of randomly initialized generated channel $H$ and $F$ matrices and all noisy received packets $X_{k}$ for $k=1, \ldots, K$. Then we compute $Y_{k}=P_{k}^{t} X_{k}, k=$ $1, \ldots, K$ where $X_{k}$ is related to the received signals for the $k$ th packet. Then, because of having QPSK symbols in $P_{k}$ we normalize all elements of the estimated $P_{k}$ matrix for $k=$ $1, \ldots, K$. Therefore, instead of applying normal PARAFAC to decompose tensor $Y$ we can have a simpler LS optimization method for estimating $H$ and $F$.

The proposed PARAFAC2 detection can be summarized as follows.

Step0. Randomly generate $H, F$.

Step1. Compute $P_{k}$ in presence of $H, F$ and fixed matrix $C$ by $P_{k}=V_{k} U_{k}^{t}$ where $F D_{k} H^{t} X_{k}^{t}=U_{k} \Delta_{k} V_{k}^{t}$ for all $k=1, \ldots, K$.

Step2. Normalize all elements of $P_{k}$ for all $k=1, \ldots, K$.

Step3. Compute $Y_{k}=P_{k}^{t} X_{k}, k=1, \ldots, K$ by $P_{k}$ s for all $k=1, \ldots, K$.

Step4. Estimate $H$ and $F$ following

$$
\begin{aligned}
& H=Y_{(1)}\left((C \odot F)^{\dagger}\right)^{\mathrm{t}} \\
& F=Y_{(2)}\left((C \odot H)^{\dagger}\right)^{\mathrm{t}}
\end{aligned}
$$

Where $(.)^{\dagger}$ stands for Moore-Penrose pseudo inverse operation, $\odot$ is Khatri-Rao product and $Y_{(n)}$ is unfolded version of tensor $Y$ on mode $n$.
Step5. Evaluate $\sigma$ as in (5). If $\left(\sigma^{\text {old }}-\sigma^{\text {new }}\right)>\epsilon \sigma^{\text {old }}$ for some small value $\epsilon$, repeat Step1, else exit.

It is interesting that after convergence the overall error of factorization is proportional to the noise level and it can be used to estimate the input $S N R$ as well.

The estimated sources using the above method are subjected to permutation and also scaling ambiguities. We used m-sequence (pseudorandom binary sequence) training sequences as pseudo information attached to the sources to solve the permutation and scaling ambiguities in the estimated sources. For solving permutation problem we attached training sequence to the first data packet. The permutation matrix can be estimated by finding the rotation matrix between the estimated and major training sequences as follow:

$$
\operatorname{Pr}=\left(\hat{S}_{t} S_{t}^{\dagger}\right)^{-1}
$$

Where $\hat{S}_{t}$ is the estimated training-sequences matrix and $S_{t}$ is the original training-sequences matrix and $\operatorname{Pr}$ includes both permutation and scaling information. Having $\operatorname{Pr}$ we are able to mitigate the permutation problem in the entire user data by:

$$
\breve{\hat{S}}=\operatorname{Pr} \hat{S}
$$

Where $\hat{S}$ is the permuted estimated user data and $\breve{\hat{S}}$ is the non-permuted user data which can be applied to the demodulation stage for user message data detection. Moreover, having permutation matrix we can solve the permutation problem by PARAFAC2 decomposition as follows.

$$
\breve{\hat{H}}=\hat{H} \operatorname{Pr}^{-1}
$$

Where $\hat{H}$ is the permuted estimated fading channel and $\breve{\hat{H}}$ is the final non-permuted channel.

In low $S N R$ cases we may use a training sequence at each packet and also applying a thresholding filter at each row of $\mathrm{Pr}$ in order to have a more accurate permutation matrix.

In this paper in order to evaluate the PARAFAC2 based MIMO detection method we compared our results with those of well known MMSE-VBLAST [8][9] MIMO detection method. MMSE-VBLAST method needs an exact fading channel and also noise power information. However PARAFAC2 based MIMO detection method does not need to the channel and also noise information.

\section{SIMULATED RESULTS}

In this section we evaluated PARAFAC2 detection method for a MIMO system having 4 transmitter and 4 receiver antennas for transmission of 4 uncorrelated QPSK baseband signals. We selected uncorrelated fading channels to evaluate the method. We generated channels randomly by choosing $r<10$ where $r=\lambda_{\max } / \lambda_{\min }$ and $\lambda \mathrm{s}$ are the eigenvalues of fading channel. We assumed that the fading channel is quasi-static during the transmission of the four 
(a)

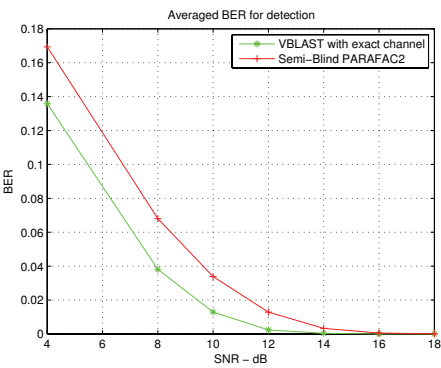

(b)

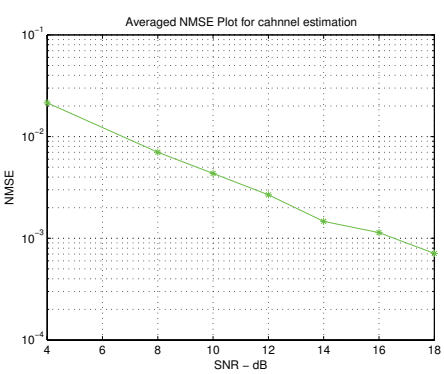

Fig. 1. (a). Averaged BERs for Semi-Blind PARAFCAC2 and MMSE-VBLAST detection methods. (b). Averaged NMSE channel estimation Error for Semi-Blind PARAFCAC2 method.

packets and each packet (with burst length equal to 1000) contains 1000 symbols including $31 \mathrm{~m}$-sequence training symbols and 969 QPSK user data symbols. We applied both MMSE-VBLAST and PARAFAC2 detection methods to detect the transmitted signals for different $S N R$ s and 100 times for each $S N R$. For MMSEVBLAST case we applied the exact channel and noise information for having the best $B E R$ as a reference to compare. Moreover, we used the estimated channel of PARAFAC 2 method and calculated the normalized mean square error $(N M S E)$ for each estimated channel by using

$$
N M S E=((\|\breve{\hat{H}}-H\|) /\|H\|)^{2}
$$

where $\|$.$\| stands for Frobenius norm. We repeated the measurement$ of NMSE for different $S N R$ s and 100 times for each $S N R$.

The results for comparing $B E R$ s of MMSE-VBLAST detection method using exact channels and our semi-blind method are shown in Figure 1(a). Obviously it can be seen that the difference between the results is relatively small, specially at $S N R$ s greater than $12 \mathrm{~dB}$. Also, the results for channel estimation show that in the worst case where $S N R=4 d B$ the channel estimation error is close to 2 percent. The results for averaged $N M S E$ at different $S N R$ s are shown in Figure 1(b).

\section{CONCLUSIONS}

In this paper we introduced a tensor factorization method for simultaneous MIMO detection and channel estimation for uncorrelated the fading channels. We used PARAFAC2 optimization method for estimating the transmitted signal and fading channel. We used msequence training symbols known to both transmitter and receiver for solving permutation and scaling ambiguities. It is shown that this method estimates the transmitted signals and the fading channel simultaneously. We evaluated the method by comparing its results using the well known MMSE-VBLAST detection method. It can be seen that the difference between the $B E R \mathrm{~s}$ of the two methods is not considerable specially for $S N R$ s greater than $12 \mathrm{~dB}$. MMSEVBLAST method needs an exact channel and also noise information. PARAFAC2 based MIMO detection method does not need the channel or noise information and it performs both signal detection and channel estimation blindly. Moreover this method estimates both the transmitted data and the fading channel in each iteration. Also we tested the method for some semi-correlated fading channels as well and the results show that this method like many other methods (e.g. VBLAST) is sensitive to rank deficiency of the fading channels.

\section{REFERENCES}

[1] M. A. Jensen and J. W. Wallace, "A review of antennas and propagation for MIMO wireless communications," Antennas and Propagation, IEEE Transactions on, vol. 52, no. 11, pp. 2810-2824, Nov. 2004.

[2] A. Jagannatham and B.D. Rao, "A semi-blind technique for MIMO channel matrix estimation,” June 2003, pp. 304-308.

[3] K.S. Bialkowski and A. Postula, "Investigations into a semiblind channel estimation technique for 2x2 MIMO system," Antennas and Propagation Society International Symposium, pp. 3257-3260, June 2007.

[4] R. Bro, "PARAFAC. tutorial and applications," Chemometrics and Intelligent Laboratory Systems, vol. 38, no. 2, pp. 149 171, 1997.

[5] N. D. Sidiropoulos, G. B. Giannakis, and R. Bro, "Blind PARAFAC receivers for DS-CDMA systems," Signal Processing, IEEE Transactions on, vol. 48, no. 3, pp. 810-823, March. 2000

[6] André L. F. de Almeida, Gérard Favier, and Jo ao C. M. Mota, "Multiuser MIMO system using block space-time spreading and tensor modeling," Signal Processing, vol. 88, no. 10, pp. 23882402,2008 .

[7] H. A. L. Kiers, J. M. F. Ten Berge, and R. Bro, "PARAFAC2 - Part I. A direct fitting algorithm for the PARAFAC2 model," Journal of Chemometrics, vol. 13, pp. 275-294, 1999.

[8] J. Benesty, Yiteng Huang, and Jingdong Chen, "A fast recursive algorithm for optimum sequential signal detection in a blast system," Signal Processing, IEEE Transactions on, vol. 51, no. 7, pp. 1722-1730, July 2003.

[9] P. W. Wolniansky, G. J. Foschini, G. D. Golden, and R. A. Valenzuela, "V-blast: an architecture for realizing very high data rates over the rich-scattering wireless channel," Signals, Systems, and Electronics, 1998. ISSSE 98. 1998 URSI International Symposium on, pp. 295-300, 1998. 